\title{
Clinical, radiological, and therapeutic features of pleomorphic xanthoastrocytoma: report of three patients and review of the literature
}

\author{
Dirk Van Roost, Rudolf Kristof, Josef Zentner, Helmut K Wolf, Johannes Schramm
}

Department of

Neurosurgery

D Van Roost

R Kristof

J Zentner

J Schramm

Department of

Neuropathology,

University of Bonn,

Germany

Correspondence to:

Dr Van Roost.

Received 13 April 1995 and in final revised form 29 January 1996

Accepted 9 February 1996

\begin{abstract}
Two out of three patients with pleomorphic xanthoastrocytoma were initially misdiagnosed and correctly interpreted only at tumour recurrence, with progression to malignancy in one. The third patient presented with a remarkably long history of epilepsy. Pleomorphic xanthoastrocytoma is a low grade astrocytoma that is still confused with other tumours. Because pleomorphic xanthoastrocytoma can become malignant even after many years of benign behaviour, a long term follow up is necessary.
\end{abstract}

(F Neurol Neurosurg Psychiatry 1996;60:690-692)

Keywords: pleomorphic xanthoastrocytoma; glioma; brain neoplasm

Pleomorphic xanthoastrocytoma, a low grade astrocytoma that mainly affects patients younger than 30, manifests itself by seizures in $75 \%$ of the patients and is still confused with other neoplasms. We report one patient who presented with a remarkably long history of epilepsy and two other patients who were initially misdiagnosed and correctly interpreted only at tumour recurrence, with progression to malignancy in one.

\section{Patient 1}

A 44 year old man presented with a 32 year history of epilepsy and no other clinical manifestation. Seizures were generalised for the first five years, ceased under medication for seven years, and took a complex partial character thereafter. Magnetic resonance imaging disclosed a left temporopolar and superficial polycystic lesion, measuring $2 \mathrm{~cm}$, with a slightly hyperintense nodule and faint enhancement (fig 1). A tumorous infiltrate of the arachnoid was not obvious. Invasive EEG recording showed a left temporal focus and herein favoured the hippocampus. A two thirds temporal lobe resection with hippocampectomy was performed. Histologically, pleomorphic xanthoastrocytoma with leptomeningeal spread was diagnosed. The resected hippocampus showed focal glial dysplasia. Three months after surgery MRI showed no evidence of residual or recurrent tumour. The patient was well and seizure free under continued antiepileptic medication.

\section{Patient 2}

A 15 year old girl presented with a three month history of epilepsy without other clinical signs. T1 weighted MRI disclosed an isointense but enhancing, left temporomesial lesion of $2.5 \mathrm{~cm}$. The adjacent arachnoid also showed enhancement. At surgery, a clear demarcation from the brain tissue was lacking. Histologically, a ganglioglioma was diagnosed. Nine months later MRI showed a recurrence
Figure 1 MRI of patient 1 showing a polycystic lesion of about $2 \mathrm{~cm}$ in diameter at the surface of the left temporal pole with a ring shaped enhancement. T1 weighted image before (top) and afier (bottom) gadolinium contrast. 


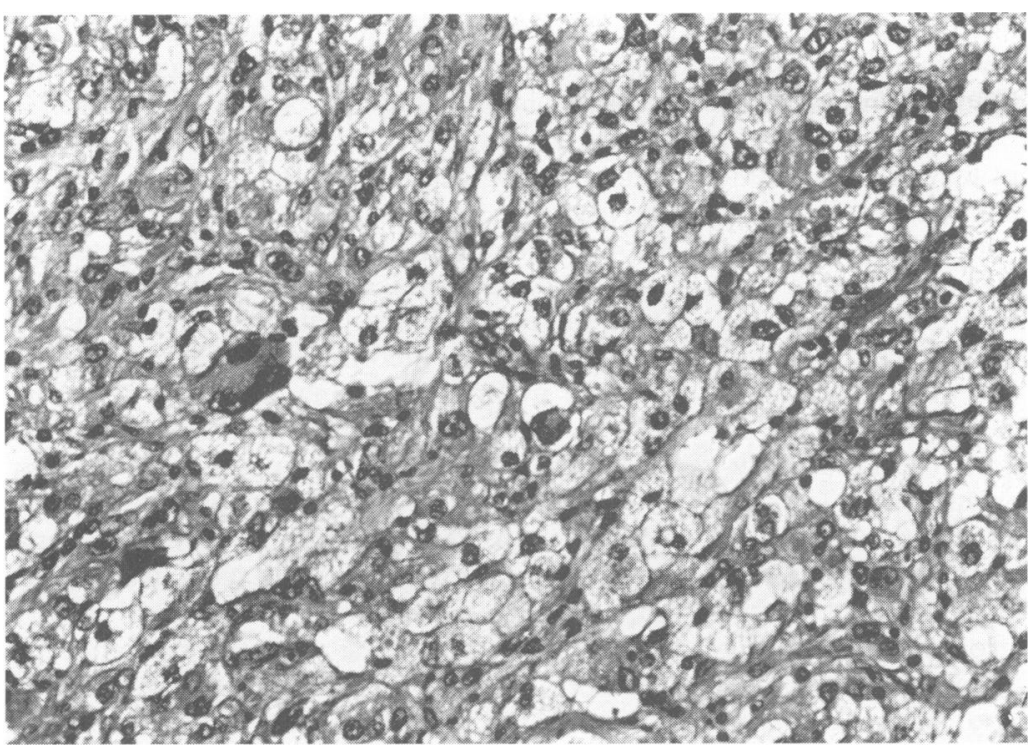

Figure 2 Photomicrograph of pleomorphic xanthoastrocytoma. Note the pronounced pleomorphism of astrocytic cells with large hyperchromatic nuclei and the presence of numerous foamy histiocytes (haematoxylin and eosin $\times 240$ ).

of $4 \times 2 \mathrm{~cm}$ along the medial and inferior resection borders, exhibiting the same signal pattern as initially. The tumour was resected again. The leptomeningeal covering of both the temporomesial region and the adjacent brainstem seemed to be infiltrated but had to be left in place, because of perforating arteries contained in it. The histological diagnosis was that of a malignant pleomorphic xanthoastrocytoma (World Health Organisation (WHO) grade 3 ). Re-evaluation of the previous specimens also confirmed the diagnosis of pleomorphic xanthoastrocytoma. No adjuvant therapy was performed. Eight months after the second operation MRI showed no evidence of recurrence.

\section{Patient 3}

Presenting with seizures, this young woman was first operated on at the age of 15 for a neoplasm initially classified as an atypical menin-

Table 1 Pleomorphic xanthoastrocytoma features reviewed from the medical literature

\begin{tabular}{|c|c|c|c|}
\hline Sex ratio $(n=87)$ : & & MRI findings $(n=25)$ : & \\
\hline Male & $53 \%$ & Hypo or isointense $\mathrm{T} 1$ & $94 \%$ \\
\hline Female & $47 \%$ & Hyperintense $\mathrm{T} 1$ & $6 \%$ \\
\hline Age $(y)$ & & Gd enhancement & $100 \%$ \\
\hline Average & $17 \cdot 5 \mathrm{y}$ & Hyperintense T2 & $100 \%$ \\
\hline Range & $3-66 y$ & Cystic appearance & $52 \%$ \\
\hline Presenting symptoms $(n=80)$ & & Perifocal oedema & $36 \%$ \\
\hline Seizures & $74 \%$ & Intraoperative findings $(n=52)$ : & \\
\hline Raised ICP & $15 \%$ & Intimate meningeal contact & $60 \%$ \\
\hline Headache & $10 \%$ & Superficial location & $36 \%$ \\
\hline Paraparesis & $1 \%$ & deep location & $4 \%$ \\
\hline Location $(n=88)$ : & & Borders well recognisable & $71 \%$ \\
\hline Temporal lobe & $61 \%$ & Borders partially recognisable & $13 \%$ \\
\hline Parietal lobe & $11 \%$ & Borders poorly recognisable & $16 \%$ \\
\hline Frontal lobe & $9 \%$ & Cystic component & $77 \%$ \\
\hline Occipital lobe & $5 \%$ & Firm consistence & $80 \%$ \\
\hline Basal ganglia & $6 \%$ & Follow up $(n=58)$ : & \\
\hline Combined supratentorial & $6 \%$ & Average duration & $7 \cdot 5 \mathrm{y}$ \\
\hline Cerebellum & $1 \%$ & Surgery only & $55 \%$ \\
\hline Thoracic spine & $1 \%$ & Mean follow up & $5 \cdot 5 y$ \\
\hline CT findings $(n=52)$ : & & Lethal tumour progression & $6 \%$ \\
\hline Hypodense & $58 \%$ & Surgery + radiotherapy & $39 \%$ \\
\hline Isodense & $38 \%$ & Mean follow up & $7 \cdot 0 \mathrm{y}$ \\
\hline Hyperdense & $4 \%$ & Lethal tumour progression & $5 \%$ \\
\hline Contrast enhancement & $95 \%$ & Surgery + radiotherapy & \\
\hline Cystic appearance & $54 \%$ & + chemotherapy & $6 \%$ \\
\hline Calcification & $11 \%$ & Reintervention & $38 \%$ \\
\hline Perifocal oedema & $31 \%$ & Mean interval & $7 \cdot 0 \mathrm{y}$ \\
\hline & & Malignancy at reintervention & $15 \%$ \\
\hline & & Tumour related deaths & $15 \%$ \\
\hline
\end{tabular}

gioma that covered the floor of the left middle cranial fossa and filled out the temporal ventricle horn. Ten years later, although the patient still presented occasional seizures without any other clinical sign, MRI showed tumour recurrence at the posterosuperior and anteroinferior borders of the resection cavity. Two firm tumour nodules were extirpated; there was no infiltration of the arachnoid. Histologically, pleomorphic xanthoastrocytoma was diagnosed. Re-evaluation of the previous specimens confirmed the diagnosis. The postoperative course was uneventful; a clinical follow up is not yet available.

Pleomorphic xanthoastrocytoma was described as a novel clinicopathological entity by Kepes $e t a l^{1}$ in 1979 . It is a glioma composed of a pleomorphic population of small and large to giant, sometimes multinucleated, cells with abundant cytoplasmatic lipid droplets and a large amount of reticulin fibres, which are partially organised to basal membranes and surround individual cells or groups of cells. According to its usually superficial location with attachment to the leptomeninges and the production of abundant reticulin fibres, pleomorphic xanthoastrocytoma is believed to originate from subpial astrocytes. Despite pronounced pleomorphism-which otherwise suggests malignancy-mitosis and endothelial proliferation are rare, necrosis is absent, and the tumour displays a relatively favourable biological behaviour. ${ }^{1}$

A literature survey covering 46 papers yielded 88 different cases of pleomorphic xanthoastrocytoma that fulfill the histological criteria of Kepes et $a l,{ }^{1}$ hence excluding those tumours with a notable amount of necrosis. ${ }^{1-11}$ Pleomorphic xanthoastrocytomas are rare tumours: five were identified by reviewing the slides of 50 patients under the age of 30 years who had had an initial diagnosis of glioblastoma or atypical glioma. ${ }^{2}$ The table summarises the clinical, radiological, and therapeutic data from the medical literature. Imaging usually shows a superficially located, often cystic tumour with a solid, enhancing nodule and little perifocal oedema. Evidence of meningeal infiltration could be a strong diagnostic argument, because meningeal infiltration is much less common in other gliomas, with the exception of the pilocytic astrocytoma.

One of our patients presented with a history of epilepsy that surpasses the longest reported duration of medical history of pleomorphic xanthoastrocytoma by 14 years. ${ }^{3}$ In patient 3 , the diagnosis of an atypical meningioma was put forward in 1981, two years after the first description of pleomorphic xanthoastrocytoma, and revised 10 years later. The tumour in patient 2 was initially interpreted as a ganglioglioma in a typical location, but revised at recurrence only nine months later, now as a malignant pleomorphic xanthoastrocytoma. We explain these misdiagnoses by an insufficient acquaintance with the novel entity and by a sampling failure.

Pleomorphic xanthoastrocytoma has also been confused with neoplasms such as fibrous 
xanthoma (fibrous histiocytoma) of the meninges, giant cell astrocytoma with histiocytic infiltration, and lipidised giant cell glioblastoma multiforme. ${ }^{124}$ Until recently, the hypothesis has been put forward that pleomorphic xanthoastrocytoma may represent a mesodermal, rather than an astrocytic, neoplasm. ${ }^{5}$ However, there is overwhelming evidence for its astrocytic nature. The tumour invariably exhibits immunoreactivity for glial fibrillary acid protein and can evolve towards a malignant glioma. ${ }^{1256}$ These findings led to the inclusion of pleomorphic xanthoastrocytoma as a clinicopathological entity in the recently revised WHO classification of brain tumours. ${ }^{12}$ One remarkable case is a patient with temporal pleomorphic xanthoastrocytoma, in whom a second, frontal pleomorphic xanthoastrocytoma developed with a latency of a few years, showing no signs of malignancy, obviously a multilocular manifestation. $^{7}$ Recently, the first pleomorphic xanthoastrocytoma in a case of neurofibromatosis type 1 has been reported. ${ }^{8}$ Within comparable follow up periods, it is surprising to find a similar number of survivors among patients who initially had an incomplete tumour resection and those who had a complete resection. This is best explained by favourable biological tumour behaviour which extends over a period of time that exceeds the considered follow up and thus determines the outcome even of incompletely resected tumours. ${ }^{9}$ The relatively benign biological behaviour of pleomorphic xanthoastrocytoma is also reflected by DNA-cytofluorometric data. ${ }^{10}$ As good clinical relief and long courses are found after subtotal surgery, radical surgery should not be sought for at the price of heavy neurological deficit.

Pleomorphic xanthoastrocytoma-like tumours with necrosis exhibit a clearly less favourable prognosis. This justifies the definition of pleomorphic xanthoastrocytoma as a tumour without any considerable amount of necrosis, mitotic activity, or endothelial proliferation. On the other hand, pleomorphic xanthoastrocytoma may undergo progression to a necrotising malignant glioma. In this regard, pleomorphic xanthoastrocytoma and pleomorphic xanthoastrocytoma-like tumours with necrotic changes could represent a single tumour entity, diagnosed at different biological stages. Pleomorphic xanthoastrocytoma that has become malignant exhibits a variable loss of lipidisation and pleomorphism, and often presents as a malignant astrocytoma or glioblastoma of the small cell type. ${ }^{126}$ Inversely, however, not all malignant gliomas that histologically exhibit lipidisation, are derived from pleomorphic xanthoastrocytoma. About $38 \%$ of the patients were reoperated on because of recurrence after an average course of seven years. Of these patients, $41 \%$ showed a progression of pleomorphic xanthoastrocytoma to malignant glioma. Because pleomorphic xanthoastrocytoma can become malignant even after many years of benign behaviour, long term follow up is necessary. There is no obvious difference in outcome between those patients who were treated by surgery alone and those treated by surgery plus radiotherapy. The value of radiotherapy therefore remains unclear and the inclination is to restrict postoperative radiotherapy to cases of recurrent pleomorphic xanthoastrocytoma that histologically present as a malignant glioma.

1 Kepes JJ, Rubinstein LJ, Eng LF. Pleomorphic xanthoastrocytoma: a distinctive meningocerebral glioma of young
subjects with relatively favorable prognosis. A study of 12 cases. Cancer 1979;44:1839-52.

2 Heyerdahl Strøm E, Skullerud K. Pleomorphic xanthoastrocytoma: report of 5 cases. Clin Neuropathol 1983;2: 188-91.

3 Palma L Maleci A, Di Lorenzo N, Lauro GM. Pleomorphic xanthoastrocytoma with 18-year survival. Case report. 7 Neurosurg 1985;63:808-10.

4 Whittle IR, Gordon A, Misra BK, Shaw JF, Steers AJW. Pleomorphic xanthoastrocytoma. Report of four cases. $\dot{f}$ Neurosurg 1989;70:463-8.

5 Paulus W, Peiffer J. Does the pleomorphic xanthoastrocytoma exist? Problems in the application of immunological techniques to the classification of brain tumors. Acta Neuropathol 1988;76:245-52.

6 Kepes J, Rubinstein LJ, Ansbacher L, Schreiber DJ. Histopathological features of recurrent pleomorphic xanthoastrocytomas: further corroboration of the glial nature of this neoplasm. A study of 3 cases. Acta Neuropathol 1989;78:585-93.

7 Iwaki T, Fukui M, Kondo A, Matsushima T, Takeshita I. Epithelial properties of pleomorphic xanthoastrocytomas determined in ultrastructural and immunohistochemical determined in ultrastructural and immuno

8 Özek MM, Sav A, Pamir MN, Özer AF, Özek E, Erzen C. Pleomorphic xanthoastrocytoma associated with Recklinghausen neurofibromatosis. Childs Nerv Syst Recklinghausen
1993;9:39-42.

9 Macaulay RJB, Jay V, Hoffman HJ, Becker LE. Increased mitotic activity as a negative prognostic indicator in pleomorphic xanthoastrocytoma. $\mathscr{F}$ Neurosurg 1993;79:761-8.

10 Hosokawa Y, Tsuchihashi Y, Okabe H, et al. Pleomorphic xanthoastrocytoma. Ultrastructural, immunohistochemical, and DNA cytofluorometric study of a case. Cancer 1991;68:853-9.

11 Herpers MJHM, Freling G, Beuls EAM. Pleomorphic xanthoastrocytoma in the spinal cord. Case report. $f$ Neurosurg 1994;80:564-9.

12 Kleihues P, Burger PC, Scheithauer B. Histological typing of tumours of the central nervous system. World Health tumours of the central nervous system. World Health Berlin: Springer, 1993. 